\title{
The Confessions of Rousseau revisited in the contemporary mode
}

\section{[Les Confessions de Rousseau revisitées en mode contemporain]}

\author{
Bahia Zemni
}

DOI: 10.18355/XL.2021.14.04.12

\begin{abstract}
The studies on Rousseauist work seem to have said it all, yet an apparently innocuous fact has rekindled our curiosity and prompted us to take another reading of the Confessions by trying to grasp the vector of singularity in its multiple dimensions, which give the Confessions the status of constituent speeches within the meaning of D. Maingueneau. We will analyze our corpus through the procedures of discourse analysis, also using lexicometry. Our intention is not in analytical completeness but to decipher what from 1782 to the present day still arouses much passion and debate.
\end{abstract}

Key words: constituent discourse- ethe- autobiography, metadiscourse, lexicometry

\section{Résumé}

Les études sur l'œuvre rousseauiste semblent avoir tout dit, pourtant un fait apparemment anodin a ravivé notre curiosité et nous a incitée à reprendre une autre lecture des Confessions de Jean-Jacques Rousseau en tentant de saisir le vecteur de singularité dans ses multiples dimensions qui donnent aux Confessions le statut de discours constituants au sens de D. Maingueneau. L'analyse de notre corpus se fera à la lumière des travaux menés dans le cadre de l'analyse du discours, en recourant également à la lexicométrie. Sans prétendre à l'exhaustivité analytique, notre objectif consiste à décrypter ce qui de 1782 à nos jours suscite encore bien des passions et débats.

Mots clés : discours constituants- éthe- autobiographie, méta-discours, lexicométrie

\section{Introduction}

Le succès de certaines séries, avec en toile de fond la philosophie, atteste de l'intérêt des jeunes et de leurs aînés à des questions essentielles du sens de la vie. La philosophe Sandra Laugier (2019: 54) avance ceci «ce qui permet de penser la morale autrement, c'est de dire que ce n'est pas seulement une question de bien ou de mal, mais aussi de perfectionnement individuel, ce qu'on appelle 'le perfectionnement moral '». L'idée qu'une série télévisée puisse servir de tremplin à asseoir une base en philosophie nous paraît séduisante en ce qu'elle introduit un air de jeunesse dans un répertoire réputé réservé aux moins jeunes. Ainsi, la série La Faute à Rousseau, en reprenant à chaque épisode une notion de philosophie, a le mérite de mettre à jour des thématiques revisitées pour en tirer des leçons de vie pour chacun des protagonistes. Thibaut De Saint Maurice (2011) de surenchérir : les séries forment un espace de pensée où se fabrique de la philosophie. Et même si la philosophie n'est pas annoncée de prime abord, elle en reste un élément essentiel en constituant ce que Gilligan (1982 : 64) nomme L'éthique du care définie par Joan Tronto (2009 : 32) comme suit «Activité caractéristique de l'espèce humaine, qui recouvre tout ce que nous faisons dans le but de maintenir, de perpétuer et de réparer notre monde. Ce monde comprend nos corps, nos personnes et notre environnement, tout ce que nous cherchons à relier en un réseau complexe en soutien à la vie. » En tendant vers le perfectionnement moral via le care, les héros de ces séries se projettent comme modèles en philosophie morale. Or, la morale, c'est d'essayer d'être soi-même. Et même si ce n'est pas évident, être soi-même, c'est arriver à trouver une voie, une forme d'authenticité, arriver à se construire comme personnage. Tel a été le but déclamé des Confessions de 
Rousseau que nous nous proposons d'étudier, en essayant de voir comment cette construction d'entreprise s'est constituée en discours constituant au sens que lui donne Maingueneau et certainement comme élément principiel de cette éthique du care. Le discours constituant étant une notion introduite par Maingueneau et Cossuta (1995, 2002 : 28) pour délimiter un ensemble de discours qui servent en quelque sorte de garants aux autres discours et qui, n'ayant pas eux-mêmes en amont des discours qui les valident, doivent gérer dans leur énonciation leur statut en quelque sorte 'autofondé'». " Inventer un langage aussi nouveau que mon projet» dirait Rousseau.

Notre démarche s'appuiera sur l'analyse du discours, notamment par l'étude des isotopies et concordances formant le méta-discours autobiographique dans les Confessions de JJ Rousseau. L'intention du projet annoncée dans les Préambules par le déroulé d'une écriture représentant la vie telle qu'elle a été vécue par le sujet se constate par la suite s'étant avérée une stratégie gagnante. La lexicométrie nous permettra d'identifier les marques constitutives des isotopies de cette construction autobiographique, notamment par l'examen des procédés énonciatifs conférant aux Confessions leurs valeurs de discours constituants de ce qui sera le genre autobiographique.

\section{Problématique et méthodologie}

Notre questionnement portera sur les procédés qui ont contribué à ce que de la matière de sa vie, Rousseau ait fait la matière de son œuvre. Comment ces procédés sont perçus comme un échantillon de référence auquel les autres s'attacheront ? L'analyse discursive de l'école française et l'approche quantitative nous permettront l'étude de ces procédés.

\section{Analyse}

\section{De l'entreprise aux discours constituants}

Lorsque Rousseau (1765) convoque les questions essentielles relativement à l'écriture de soi, il est déjà un écrivain chevronné avec des textes désormais célèbres, La Nouvelle Héloïse, Le Contrat social, mais surtout, il pose les jalons d'une écriture dont l'objet est le déroulé de vie. Certes Rousseau n'est pas innovateur dans cette forme d'écriture, des civilisations anciennes du Moyen Orient avec l'Apologie de Hattusili II (Mouton \& van den Hoven, 2015 : 68) ou des Confessions de Saint Augustin ont déjà produit des récits de soi mais ces derniers ne sont pas annoncés comme écrits à statut inédit.

En effet, dans sa narration assumant et assurant la sincérité, l'authenticité, la vérité, l'auteur positionne ses Confessions en outil de perfectionnement moral et les place dans ce que Philippe Lejeune appellera « Le Pacte autobiographique » En portant d'un seul coup le genre à un haut degré de perfection, Rousseau en a infléchi l'histoire : désormais quiconque entreprend d'écrire sa vie pense à Rousseau, que ce soit pour l'imiter ou le condamner. On est obligé de se situer par rapport à lui. » (1985: 81).

\subsection{Structure des Confessions}

\section{L'intitulé Les Confessions}

Sémantiquement, l'intitulé renvoie au domaine religieux en regard à l'époque où l'église était puissante et autoritaire, la notion d'aveux et/ou de péchés serait alors envisageable. Cependant, ses apostrophes aux lecteurs laissent entrevoir une autre signification, celle de vouloir s'expliquer, de donner une image valorisante de soi et comment mieux dire « le vil et le sublime » que sur le mode des aveux ou des 
Confessions. C'est bien cette ouverture à l'autre qui annoncera le genre moderne d'autobiographie.

\subsection{L'âge d'or et l'âge des tumultes des deux parties}

L'œuvre est constituée de deux parties, chacune comportant six livres. Une première partie expliquant la structuration de son caractère, première enfance heureuse où Rousseau était lecteur précoce des œuvres classiques. Mais rapidement livré à luimême, le jeune Rousseau mène une vie instable, connait les premiers émois sensuels. Il découvre aussi l'importance des influences et leur poids dans le cours de la vie.

La seconde partie, de six livres également, est celle des rencontres avec les philosophes, les musiciens, les encyclopédistes. C'est une période d'échecs et de succès. Toutefois, des conflits et des divergences avec d'anciens amis vont installer un sentiment d'écart, de solitude. Se sentant traqué par ses semblables, il décide de réformer sa vie selon des principes de simplicité, de vertu et de frugalité. C'est une période de création avec le succès de la Nouvelle Héloïse contrebalancé par les refus retentissants de l'Emile aussi bien à Paris qu'à Genève. S'ancre alors chez Rousseau le sentiment de complot et de persécutions qui le conduiront d'errance en errance. C'est de retour à Paris qu'il écrit Les Confessions avec la seule compagnie de Bernardin de Saint Pierre.

\section{Des premiers signes aux conventions}

En s'appuyant sur les thématiques de l'amour, l'identité, la justice, l'auteur entreprend le premier jalon du pacte, à savoir «Intus, et in cute ", en épigraphe des Confessions. Ainsi le critère absolu " une personne réelle fait le récit de sa propre existence » de la définition de P. Lejeune (1998 : 34) est posé d'emblée. C'est aussi dans cet idéal moral de sincérité, promut par Rousseau et qui s'est aujourd'hui intégré aux valeurs collectives allant jusqu'à en imprégner les séries télévisées que l'entreprise s'élabore par la narration introspective. L'instance énonciative est donc déterminée, le narrateur (l'instance qui dit Je), le personnage (le $J e$ dont il est question) et l'auteur (le producteur du texte) sont parfaitement analogues.

Cette instance à strates génère une relation embrayée qui demande à la croire, à l'estimer, à l'admirer et à l'aimer. Le retour au passé sous le mode de l'authenticité, « je veux montrer à mes semblables un homme dans toute la vérité de la nature, et cet homme, ce sera moi » est constitutif d'un méta-discours superposant expériences vécues et commentaires personnels. "C'est ici de mon portrait qu'il s'agit et non pas d'un livre. Je vais travailler pour ainsi dire dans la chambre obscure : il n'y faut point d'autre art que de suivre exactement les traits que je vois marqués. Je prends donc mon parti sur le style comme sur les choses. Je ne m'attacherai point à le rendre uniforme ; j'aurai toujours celui qui me viendra, j'en changerai selon mon humeur, je dirai chaque chose comme je la sens, comme je la vois, sans recherche, sans gêne, sans m'embarrasser de la bigarrure.» Rousseau (1764). L'utilisation du présentatif «c'est ici » donne une valeur de vérité générale au «portait »; le futur de certains verbes « attacherai, aurai, changerai, dirai, associé à l'anaphore «sans $\times 3$ » amplifie la promesse d'évacuer toute recherche esthétique. Rendue solennelle, cette promesse est prise en charge par le champ lexical de la description sans artifice « En me livrant à la fois au souvenir de l'impression reçue et au sentiment présent je peindrai doublement l'état de mon âme, savoir au moment où l'événement m'est arrivé et au moment où je l'ai décrit ; mon style inégal et naturel, tantôt rapide et tantôt diffus, tantôt sage et tantôt fou, tantôt grave et tantôt gai fera lui-même partie de mon histoire ».La reprise anaphorique « tantôt » traduit avec emphase la volonté de se dire tel quel, de se dévoiler. 
Cette volonté du dévoilement se manifeste par un «je » partagé entre son sentiment intérieur et les regards des autres sur lui. «J'ai dit le bien et le mal avec la même franchise, je n'ai rien tu de mauvais, rien ajouté de bon». Tout au long des Confessions, nous retrouvons ce parcours isotopique du bien et du mal, habile stratégie énonciative conférant une construction d'éthos. L'aveu des péchés à connotation symbolique aux Confessons de Saint Augustin se déroule en plaidoyer de soi. «Je me suis montré tel que je fus, méprisable et vil quand je l'ai été, bon, généreux, sublime quand je l'ai été », le pronom J'octroie à l'énonciateur « la figure d'un garant doué de propriétés physiques (corporalité) et psychologiques (caractère) en s'appuyant sur un ensemble diffus de représentations sociales évaluées positivement ou négativement, de stéréotypes que l'énonciation contribue à transformer » Maingueneau (2014: 21). Cette énonciation provoque la construction d'un éthos qualifié en analyse du discours d'éthos discursif. Ducrot (1984: 22) décline cette notion en éthos dit et éthos montré. L'éthos discursif se montre dans l'acte d'énonciation, l'éthos dit porte sur les propriétés de l'énonciation elle-même, ce que l'énonciateur dit sur lui-même, «je veux montrer à mes semblables un homme dans toute la vérité de la nature, et cet homme ce sera moi » " ma parole est sévère ", et l'éthos montré, ce que montre sa manière de l'énoncer. Nous allons étudier dans les parties suivantes la stratégie cohérente à travers les divers procédés qui révèlent le positionnement de l'auteur dans ses textes ainsi que ses intentions.

\section{Les Confessions à travers les prismes de l'analyse du discours et de la lexicométrie}

\section{De quelques formes pivots (c)}

Dans son assertion à se dévoiler, à ne rien taire, aux lecteurs Rousseau instaure le pacte de lecture en assurant que ce travail d'introspection est essentiellement porté par la mémoire. Et, même si des défaillances surviennent, ce ne seront que des « ornements » pour pallier à celles-ci. L'important en est l'authenticité, la vérité. A ce stade de notre travail, nous intégrons la statistique lexicale (b) qui permet d'établir des lectures tabulaires à travers l'œuvre des Confessions.

Graphe1 obtenu avec le logiciel Hyperbase 


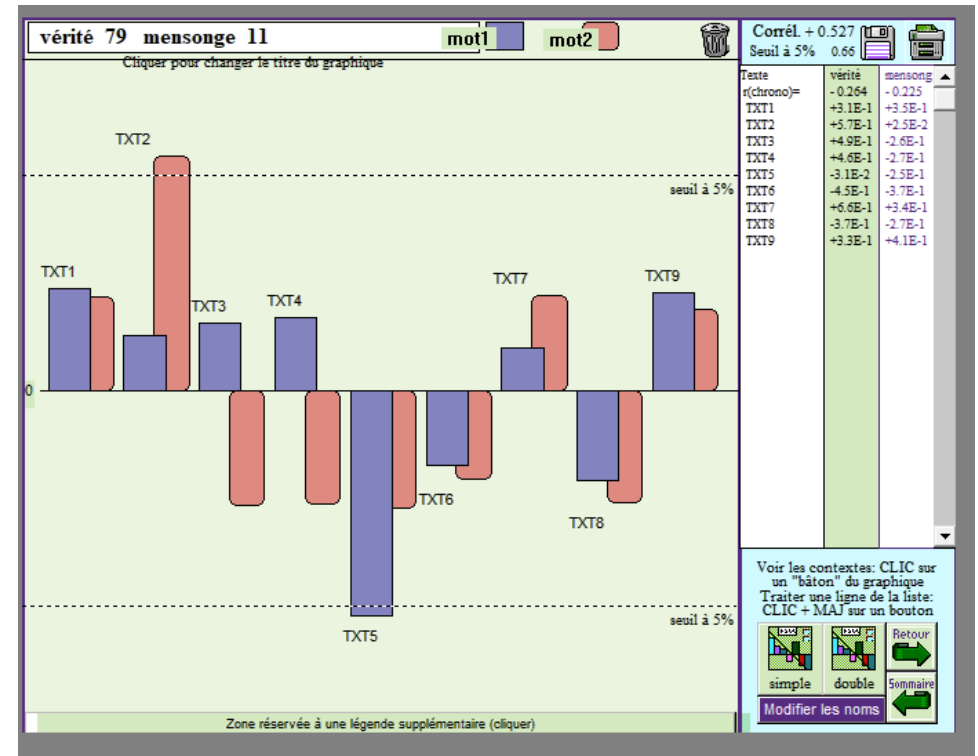

Titre du graphe I: Répartition des 2 vocables "vérité et mensonge " dans Les Confessions

A titre d'illustrations nous nous intéressons aux mots "vérité et mensonge" particulièrement évocateurs du champ lexical de l'authenticité. Le traitement informatique de texte avec le logiciel Hyperbase permet dans un ler temps le décompte de ces 2 vocables puis dans un second temps de leurs répartitions tout au long de l'œuvre. Le nombre d'itérations de « vérité » est 79 alors que mensonge » est 11 , c'est une sur-représentation de la vérité face au mensonge et donc une indication de son importance pour l'auteur. La répartition des bâtonnets au-dessus de la ligne horizontale o indique les emplacements de ces mots dans les livres. Notons que la barre la plus représentative (la plus haute) se situe dans la première partie ; c'est dire tout le désir de l'auteur d'exprimer sa vérité. Un regard plus attentif avec les contextes de ces vocables, leurs 'espaces de production' ou leurs 'espaces textuels' Cossutta et Maingueneau (2019: 6) vont nous éclairer sur son emploi dans une visée rhétorique argumentative.

Maingueneau (1990 : 26) note à propos de l'énonciation qu'il s'agit de l'ensemble des opérations qu'effectue le sujet parlant construisant un ensemble de représentations communicables dans un énoncé. Dans Les Confessions, le sujet énonciateur, en tant qu'instance désirante engagée et instance de locution formule les représentations et manifestations de soi à travers le déictique «je ». Phillipe Gasparini (2004: 166) parle d'un «je autodiégétique » qui mime soit l'énonciation orale soit l'écriture intime. Selon Danon-Boileau (2007) toute trace d'opération d'échange est foncièrement orientée vers le langage. Or, ici, la source des énoncés est marquée par le recours au terme spécifique du «je» et de ses autres manifestations qui se présenteront comme le statut de représentant métalinguistique de l'origine subjective de l'énoncé.« Je veux montrer à mes semblables un homme dans toute la vérité de la nature ». Ainsi fonctionne ce «je » qui veut s'aligner aux autres hommes en passant par la restitution de la vérité, quelle qu'elle soit. Ce qui nous pousse à regarder de plus près le voisinage linguistique de ce pronom dans le but d'en dégager les représentations visées par l'auteur.

\section{Graphe II avec Hyperbase}




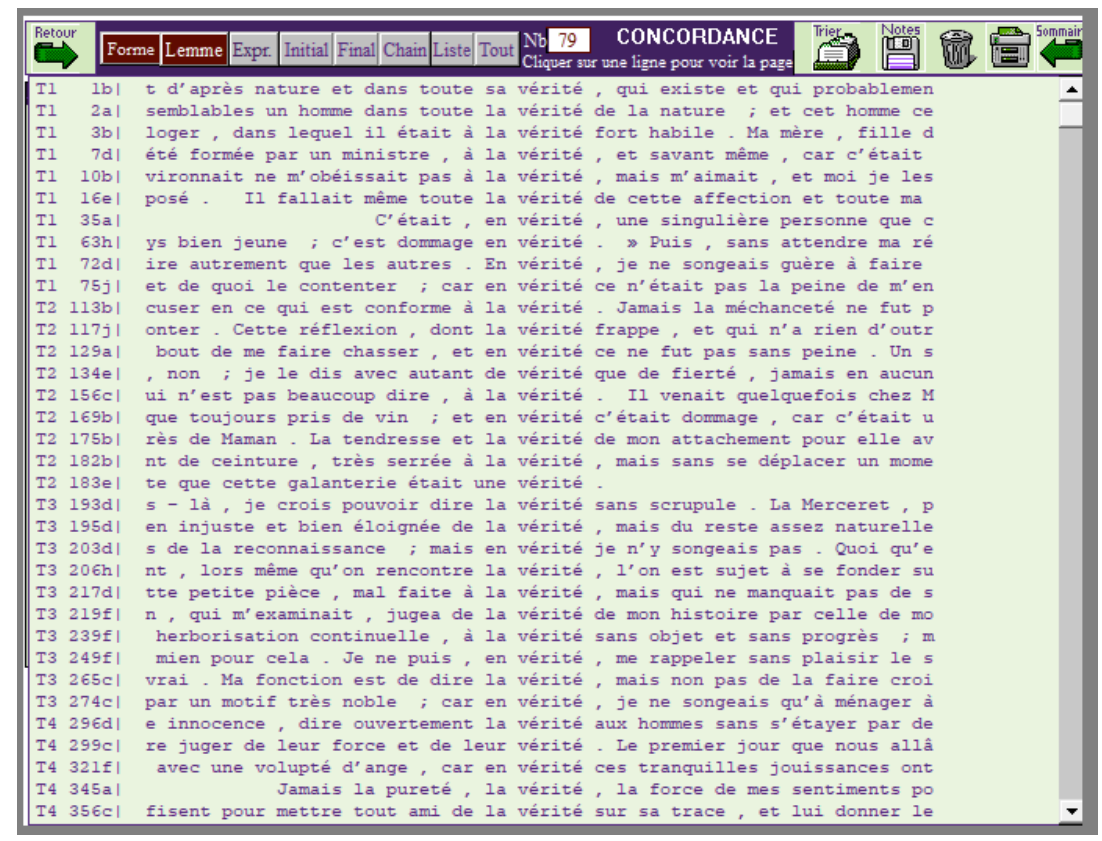

Titre du graphe II : Contexte immédiat du vocable «vérité » dans Les Confessions

La première occurrence «Voici le seul portrait d'homme, peint exactement d'après nature et dans toute sa vérité » montre un sujet d'énonciation. Ce type d'énonciation principale st repris plusieurs fois donnant les éléments de la narration avec des verbes à l'imparfait et commenté par un énonciateur somme toute indulgent. Promesse auctoriale projetant une construction d'éthos auctorial qui sera répartie en suites d'intentions pour en fabriquer les éthe !

A la ligne 12 du graphique ci-dessus, un présent de l'indicatif attire l'attention, « cette réflexion, dont la vérité frappe, m'a été d'un grand usage dans le cours de ma vie pour me faire tenir à ma place paisiblement ». Ce présent à valeur de vérité générale promet la garantie de l'humilité de l'auteur tout au long de sa vie. Ce présent signale aussi l'intention d'une communication immédiate et intime où l'effet de vérité assigne l'illusion d'un sujet écrivant par un sujet lisant. Gasparini (2004 : 208) note à propos de ce présent «Mais le rôle du présent, comme celui de la première personne, ne se limite pas à embrayer sur le mode discursif, [...] C'est en effet le temps du métadiscours, donc du commentaire du récit. Il marque une pause dans le processus narratif, pause au cours de laquelle va s'engager une réflexion sur l'instrument de la rétrospection, c'est-à-dire sur la mémoire » Les 2 autres formes « $m$ 'a et $m a$ place » viennent renforcer cette image d'humilité et interviennent dans l'appareillage intradiscursif de l'éthos montré.

Plus loin, (ligne 29) « Ma fonction est de dire la vérité, mais non pas de la faire croire », l'utilisation de l'adjectif possessif Ma indique l'éthos montré. Le présent de l'indicatif inscrit cette assertion dans la temporalité de toute l'œuvre. Nous pouvons 
dès à présent remarquer l'articulation de ces éthe contribuant à la scène d'énonciation pour en dévoiler la persuasion et la vérité.

Dans la même perspective d'analyse, nous pourrions relever les récurrences du vocable «mensonge ", l'étude des 11 occurrences révèle que la situation imposait ce mensonge plutôt comme un déni des personnages. Toutefois la phrase suivante " [...] et je crois sentir que mon aversion pour le mensonge me vient en grande partie du regret d'en avoir pu faire un aussi noir. » laisse comprendre que le sujet narrateur personnage est un fervent défenseur de la vérité. Au terme de cette partie, nous pouvons conclure que le critère de sincérité est présent dans l'œuvre et remplit une des conditions du pacte de lecture.

Le récit des événements de la vie du sujet Rousseau est proposé comme un moyen de démêler son passé, se réappropriant par la même occasion des prétextes pour de futures justifications. Un autre logiciel de lexicométrie nous permettra d'en saisir les inscriptions dans l'œuvre.

\section{Les isotopies de l'introspection}

Lors du procès organisé en Juin 2019 au Collège de France par la Fédération française de Débat et d'Éloquence ; consacré à Rousseau(d), l'écrivain scénariste Jean-Claude Carrière évoquait la difficulté à raconter le passé «la mémoire est une activité du temps présent, la mémoire étant l'exercice contemporain de ce que nous nous souvenons ». Pour illustrer cette difficulté, il a évoqué la fameuse bataille de Poitiers notée dans les manuels d'histoire, pour préciser qu'en réalité c'était une bataille perdue à Tours et carrément occultée par les historiens arabes. C'est finalement reconnaitre tout le péril de relater le passé. Rousseau le note lui-même : "J'écris absolument de mémoire, sans monuments, sans matériaux qui puissent me la rappeler. Il y a des événements de ma vie qui me sont aussi présents que s'ils venaient d'arriver, mais il y a aussi des lacunes et des vides que je ne peux remplir qu'à l'aide de récits aussi confus que le souvenir qui m’en est resté » Livre II.

Pourtant, de la matière de sa vie il en a fait la matière de son œuvre et se présente même comme un échantillon auquel les autres s'attacheront. Pour analyser ce mouvement d'introspection, nous tenterons de tracer les isotopies du temps; de la mémoire et de certaines thématiques s'y afférant. Nous adoptons la définition de l'isotopie de Français Rastier qui la désigne comme étant un procédé sémantique indiquant la présence d'un même sème dans plusieurs termes d'un texte, ce qui permet de les relier entre eux et donc de tracer un parcours de sens. "On appelle isotopie toute itération d'une unité linguistique.[...].C'est la permanence d'une base classématique hiérarchisée qui permet, grâce à l'ouverture des paradigmes que sont les catégories classématiques, les variations des unités de manifestation, variations qui, au lieu de détruire l'isotopie, ne font que la confirmer » (Rastier 1987 p 92). L'isotopie duelle du mal et du bien se décline dans les Confessions en un lexique subjectif que nous étudierons avec les logiciels quantitatifs.

\section{Graphe III avec Tropes}




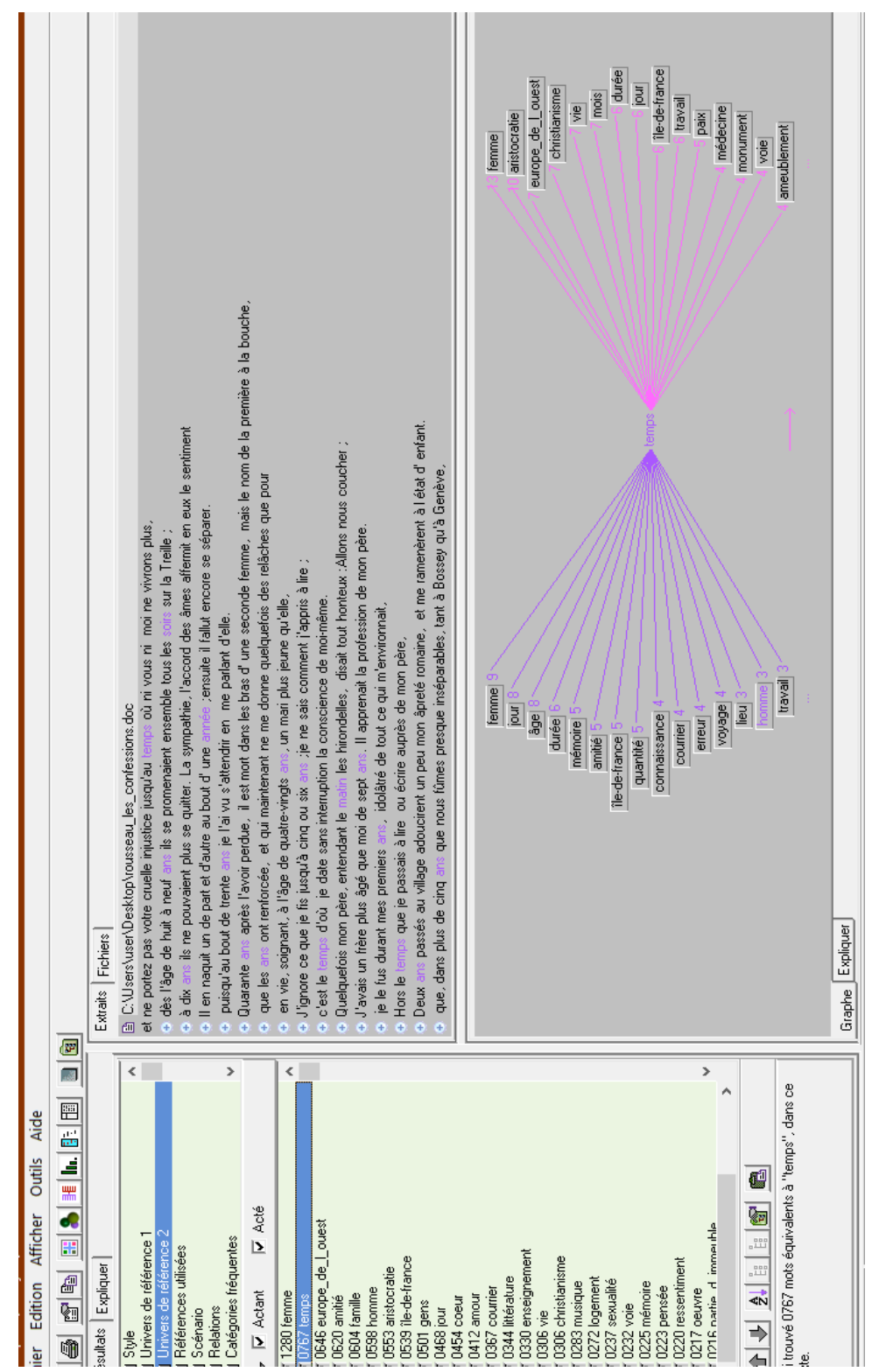

Titre du Graphe III : Forme pivot « Temps » avec les relations sémantiques

Le logiciel Tropes permet de reconstruire les parcours sémantiques des formes pivots, ce qui explique notre intérêt pour son utilisation. En effet, il permet une lecture tabulaire de ces parcours, autrement dit, quels sont les mots les plus proches de la forme pivot, ici, «temps » et le nombre de ces relations, Ainsi, nous pouvons dégager l'isotopie du temps à travers le récit de vie où les protagonistes majeurs sont, la femme, l'amitié, la connaissance, la paix. Les références aux « voyage, Iles de France, Europe de l'ouest,...» agissent dans le paradigme référentiel et assoient les 
fondements du pacte référentiel au narrateur/sujet/personnage.).Les travaux d'E. Bruss, 1974, ont permis cette référentialité associée à un «pacte de vérité » dans Signes de vie, ou d'un « engagement de sincérité » qui transcendent le texte en lui conférant un statut illocutoire, première isotopie de la sincérité et de la vérité qui entre en dualité avec celle de l'aveu et du dévoilement formant cet effet de miroir du bien et du mal que nous évoquions au début de ce travail.

Voyons les tracés sémantiques avoisinant le vocable «désespoir» dans le graphe suivant :

\section{Graphe IV avec Tropes}

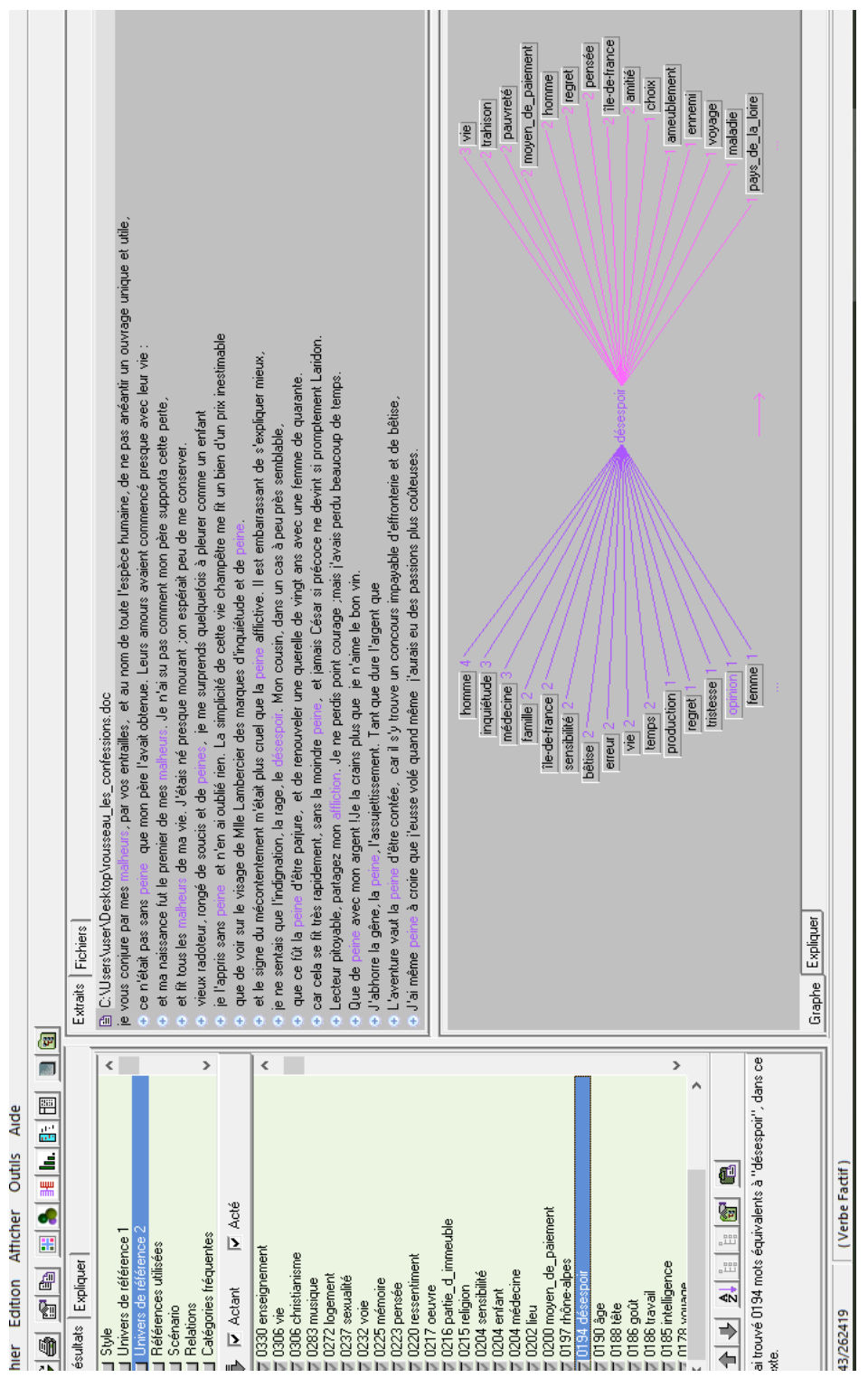


Titre graphe IV : Forme pivot « désespoir » avec les relations sémantiques

La variété des formes lexicales pour traduire le désespoir de l'auteur (hommes, inquiétude, regret, tristesse, trahison, pauvreté, ennemi, maladie) révèle l'amplitude de ce désespoir et trace cette isotopie du dévoilement. Notons la variété du vocabulaire axiologique «un de ces ennemis, cessez de l'être avec ma cendre ; d'avoir été généreux et bon quand vous pouviez être malfaisant et vindicatif » que l'auteur prend comme prétexte pour dévoiler la partie sombre de soi mais surtout pour critiquer le jeu des apparences que nécessitait la vie sociale de son temps. En utilisant un discours à dominante axiologique négative il trace un réquisitoire des travers des relations sociales.

\section{Graphe V avec Tropes}

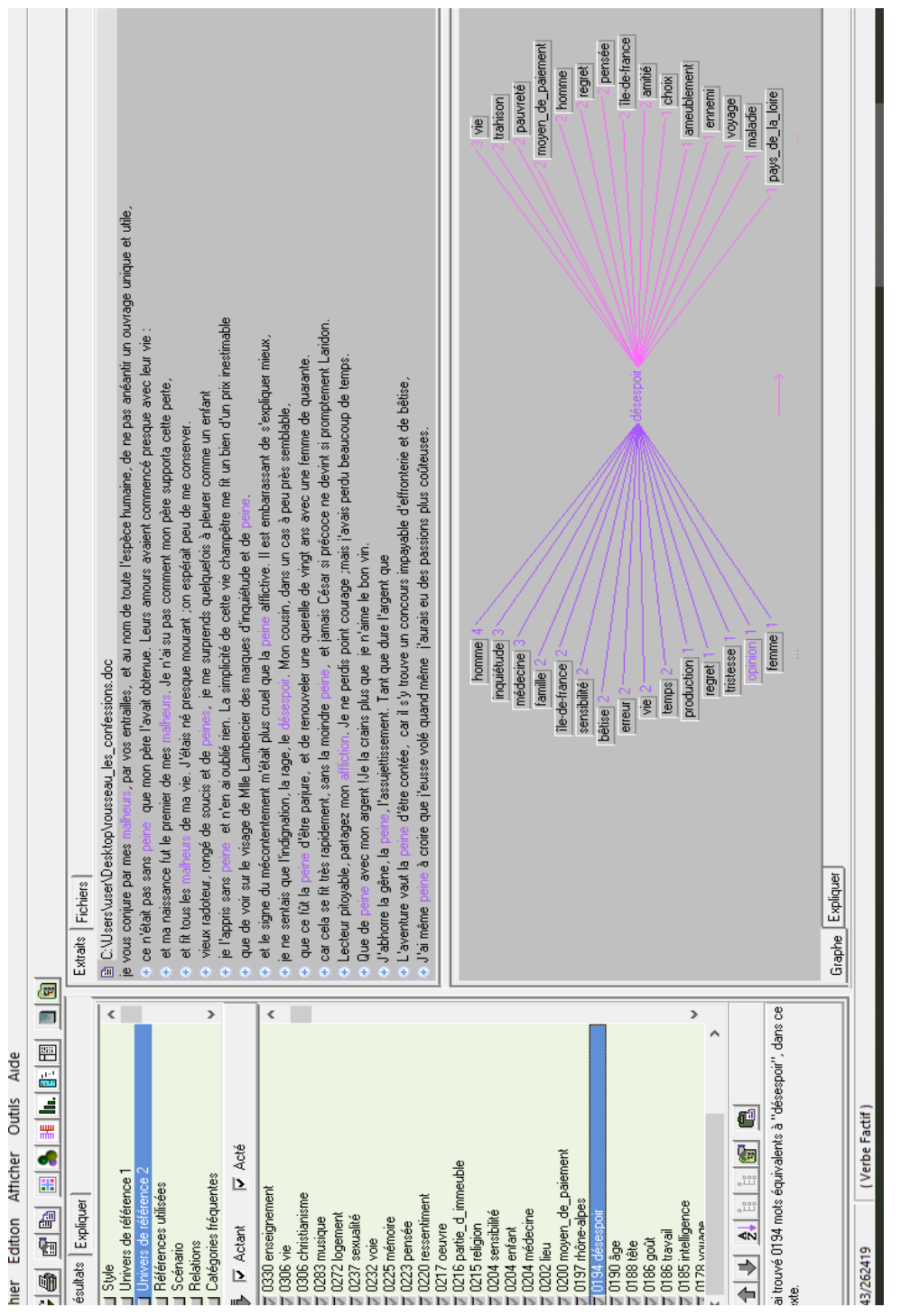

XLinguae, Volume 14 Issue 4, October 2021, ISSN 1337-8384, eISSN 2453-711X 
Titre graphe $\mathbf{V}$ : Les formes de modalisateurs

Un examen attentif des catégories fréquentes de la colonne de gauche donne les chiffres suivants obtenus avec le logiciel Tropes :

- $\quad$ Pourcentage le plus important des modalisateurs : d'opposition 19,9\% et $46,6 \%$

- $\quad$ Pourcentage le plus fréquent des adjectifs axiologiques : subjectifs $62,2 \%$

- $\quad$ Pourcentage de la catégorie des pronoms : « je » avec 57. \%

En linguistique, les modalisateurs sont les outils morphologiques, lexicaux, syntaxiques par lesquels se manifeste l'attitude de l'auteur dans son énoncé. Les Confessions sont un récit à la première personne et construisant la méta-scène du philosophe en position de victime. Rousseau l'annonce dès le début avec la phrase : «Ma naissance fut le premier de mes malheurs. » et donne une portée dramatique à sa destinée. Il serait vain de penser qu'un acte d'écriture de soi exclurait un travail esthétique ; en quête d'une construction, le sujet amené à se dire subit le décalage du temps, mais en organisant son texte à partir d'un «je » élaboré de faits factuels, associant narrations et commentaires Rousseau a bien établi la leçon de vie en phénomène d'introspection à dimension statutaire.

\section{Résultats et discussion}

Après nous être intéressés à la structure de notre corpus pour tenter d'en saisir les mouvements de pensée et de dessein de Rousseau, nous avons eu recours aux théories de l'énonciation. Notre analyse a montré un « je » qui se disait tout au long de deux isotopies, celles du bien et du mal et fonctionnant tel un chiasme pour en dégager certains critères, reconnus par la suite spécifiques de l'autobiographie. L'utilisation des logiciels lexicométriques nous a permis de dégager des formes pivots, considérés comme temps forts des isotopies avec les marques caractéristiques des discours constituants de l'autobiographie « les discours constituants [...] ils ne peuvent servir de garants aux autres discours qu'en construisant à travers leur énonciation les conditions de leur propre validité, processus qui ne fait qu'un avec leur mode d'existence. Ce travail d'autolégitimation suppose une inscription profonde dans l'interdiscours et l'élaboration de scènes d'énonciation spécifiques » (Maingueneau, $2002: 134)$

\section{Conclusion}

Boudée, ignorée ou mésestimée, l'autobiographie s'est finalement constituée en genre reconnu ayant ses marques ostentatoires. Reconnue comme un genre avec ses détracteurs et ses adeptes. Ph. Lejeune a démontré son impact au niveau des études critiques et universitaires avec la notion de «pacte de lecture » comme l'a noté Chloé Vettier (2019) dans son article Postérité du pacte autobiographique «sinon célébrer, l'entrée définitive de la notion de pacte autobiographique au panthéon des études littéraires. " Les Confessions de Rousseau adressées à Dieu ou aux hommes sont avant tout un long plaidoyer, tentant de donner une image positive de par l'aveu même de ses erreurs, fautes et justifications. Pour construire cette image de soi positive, l'auteur a eu recours à différentes stratégies, tantôt sous forme dramatique, tantôt lyrique, parfois dramatique en édifiant un ethos d'un sujet tour à tour héroïque, batailleur et serein.

En adoptant une posture de complicité vis-à-vis de son lecteur, l'auteur a bâti, sur le mode de dimension morale, un personnage en quête de comprendre le monde, de trouver une voie pour se représenter et se construire.

Nous évoquions en début de travail les séries reposant sur une forme particulière de divertissement tout en invitant les spectateurs à repenser le monde en fonction des 
problématiques nouvelles. Leurs toiles de fond philosophiques, greffées d'options sociales, morales, politiques, est à notre sens la meilleure marque de reconnaissance de l'œuvre de Rousseau. En exposant son intimité par le détour de l'extimité (e), l'auteur a déterminé un sujet validé par le regard d'autrui. Pour clore ce travail, nous nous référons à Georges May qui constatait la progression ascendante du nombre d'autobiographies publiées depuis 1782, Les Confessions annonçant une " première vraie prise de conscience collective de l'existence littéraire de l'autobiographie. » et que nous désignons « discours constituants ».

\section{Notes}

(a) https://www.youtube.com/watch?v=89VcEcsGuRI

(b) La statistique lexicale ou lexicométrie est une approche basée sur la quantification des unités linguistiques d'un corpus. Les positionnements de ces unités les unes envers les autres, de leurs distanciations ou au contraire éloignements ainsi que de leurs combinaisons forment des axes de lecture parfois inédits. La récurrence de ces phénomènes permet une lecture tabulaire et autorise des saillances, contrastes, notamment par l'obtention de graphes illustrant ces saillances Les logiciels utilisés ici, Hyperbase et Tropes permettent cette exploration quantitative de l'ensemble des livres formant Les Confessions.

(c) La forme pivot appelée aussi forme pôle est un indicateur de réseaux thématiques. En lexicométrie le mot pôle peut être assimilé au mot thème qui, lors du dénombrement lexical, signale la pertinence d'une étude des systèmes d'associations relatifs aux vocables les plus fréquents du corpus.

(d) Source : https://www.youtube.com/watch? $\mathrm{v}=89 \mathrm{VcEcsGuRI}$

(e) Terme attribué à Michel Tournier, 2004, qui définit l'extime comme étant ce qui un intime sorti de l'intériorité, un intime que le sujet espère trouver, hors de lui, dans le champ de l'autre.

\section{Acknowledgement}

This research was funded by the Deanship of Scientific Research, Princess Nourah bint Abdulrahman University through the Fast-track Research Funding Program.

\section{Bibliographic references}

ALLAMAND, C. 2018. Le «Pacte » de Philippe Lejeune ou L'autobiographie en Théorie. Paris : Edition Critique et Commentaire Paris : Honoré Champion, coll. EAN 9782745346834

BARTHES, R. 1975. Roland Barthes par Roland Barthes. Paris: Seuil.

CESPEDES, J. 2001. Le Problème ontologique de l'autobiographie. In : Cahiers de Narratologie, pp. 273-284.

COSSUTTA, F. - MAINGUENEAU, D. 2019. L'analyse du discours philosophique : Bilan et perspectives. In : Argumentation et Analyse du Discours, n. 22, pp. 1-17. Available online: http://journals.openedition.org/aad/2969 DOI : https://doi.org/10.4000/aad. 2969

DELORMAS, P. 2008. L'image de soi dans les "autographies» de Rousseau. In : Argumentation et Analyse du Discours, n. 1, pp. 1-11.

DE SAINT, M. 2009. Philosophies en Séries. Paris : Ellipses.

DESCHENES, M. 2015. L'attention aux récits sur soi, Paul Ricœur et Carol Gilligan autour du tragique freudien. In : Logoi.ph, vol.1, n. 2, pp. 207-227.

DHIFAOUI, A. 2006. Autobiographie et histoire dans Les Confessions de Rousseau. In : Autobiographie d'Ibn Kheldoun à nos jours, actes du Colloque tenu les 23, 21, 25 novembre 2006 à l'Académie des Sciences, des Lettres, et des Arts de Carthage, 
(BeitAl Hikma), textes réunis et présentés par Hédia Khadhar et Emna Beltaif, Cahiers du CERES, Série Littérature, n. 10, pp.57-81.

DOUBROVSKY, S. 1988. Autobiographique : De Corneille à Sartre. Paris : PUF.

GENETTE, G. 1972. Figures III. Paris : Seuil.

FILLIPPI-DESWELLE, C. 2014. Le sujet modal ou la construction du maître du JE en question. In : BESNAULT-LEVITA, A., Depraz, N., WINTERMEYER R (éds) 2014. Construire le Sujet. Limoges : Lambert-Lucas. Available online: https://halnormandie-univ.archives-ouvertes.fr/hal-01775104

GASPARINI, P. 2004.Est-il Je ?. Paris : Seuil.

GUSDORF, G. 1991.Les Ecritures du Moi : Tome 1. Paris : Odile Jacob.

LAUGIER, S. 2019. Nos Voies en Séries: Philosophie et Morale d'une Culture Populaire. Montpelier : Climats.

LAUNAY, L. - LAUNAY, M.1981, Le Vocabulaire Littéraire de J.-J. Rousseau. In : Dix-huitième Siècle, n. 13, Juifs et judaïsme, pp. 525-526.

LEJEUNE, P. 1975. Le Pacte Autobiographique. Paris : Seuil, coll « Poétique ».

LEJEUNE, P. 1980. Je Est un Autre : L'autobiographie de la Littérature aux Médis.

Paris : Seuil, coll « Poétique».

LEJEUNE, P. 2009. Intime, privé, public. In : La faute à Rousseau, n. 51, pp. 9-15.

LEJEUNE, P. 1998. L'autobiographie en France. Paris : Armand Colin.

MAINGUENEAU, D. 2014. Retour critique sur l'éthos. In : Langage et Société, n.149, pp. 31-48.

MAINGUENEAU, D. - CHARAUDEAU, P. 2002. Dictionnaire d'Analyse du Discours. Paris : Seuil.

MAINGUENEAU, D. - COSSUTTA, F. 1995. L'analyse des discours constituants. In Langages, n. 117, pp. 112-125.

MARSOP, P. 2010. Leçon de courage: A la frontière de la littérature et de la philosophie. In: Verbum Analecta Neolatina, vol. 7, n. 1, pp. 185-195. DOI : 10.1556/Verb.122010.1.12

MAY, G. 1979. L'autobiographie. Paris : Presses Universitaires de France.

MOUTON, A. - VAN DEN HOVEN, C. 2015. Les Noms des Témoins Divins du Traité entre le Roi Hittite Hattušili III et le Pharaon Ramsès II : Un Exemple d'Interprétation. In : Colin, Huck, F., Vanseveren S. O. (éds) 2015. Interpretatio. Traduire l'Altérité Culturelle dans les Civilisations de l'Antiquité.

Paris : De Boccard. ISBN 978-2-7018-0375-3

POULET, G. 1977. Entre Moi et Moi : Essai Critique sur la Conscience de Soi. Paris : Corti.

RASTIER, F. 1987. La Sémantique Interprétative: Formes Sémiotiques. Paris :

Presses Universitaires de France.

RICOEUR, P. 1990. Soi-même Comme un Autre. Paris : Seuil.

ROUSSEAU, J-J.1782. Les Confessions. Available online:

https://bibliothequenumerique.tv5monde.com/livre/161/Les-Confessions

ROUSSEAU, J-J. Préambule du Manuscrit de Neufchâtel. Available online: http://www.lettres.org/Confessions/rousseau_txt01.htm

SIMONET-TENANT, F. - COUDREUSE, A. 2009. Préambule. In : Itinéraires, n. 4. DOI : https://doi.org/10.4000/itineraires.583

TODOROV, T.1981. Le dernier Barthes. In : Poétique, n. 47, pp. 323-327.

TOURNIER, M. 2004.Journal Extime. Paris : Gallimard.

TRONTO, J. 2009. Un Monde Vulnérable: Pour une Politique du Care. Paris: Editions La Découverte.

VETTIER, C.2019. Postérité du pacte autobiographique. In: Acta fabula, vol. 20, n. 5.

Words: 5118

Characters: $32513(18,10$ standard pages) 
Bahia Zemni

Translation Department

College of Languages

Princess Nourah bint Abdulrahman University/Airport Road, King Khalid International Airport,

Riyadh 11564, PO Box 84428,

Saudi Arabia

baalzemni@pnu.edu.sa

ORCID: https://orcid.org/0000-0002-6238-7509 